QUALITY IMPROVEMENT REPORT

\title{
A web-based incident reporting system and multidisciplinary collaborative projects for patient safety in a Japanese hospital
}

\author{
K Nakajima, Y Kurata, H Takeda
}

Qual Saf Health Care 2005;14:123-129. doi: 10.1136/qshc.2003.008607

Problem: When patient safety programs were mandated for Japanese health care institutions, a safety culture, a tool for collecting incident reports, an organizational arrangement for multidisciplinary collaboration, and interventional methods for improvement had to be established.

Design: Observational study of effects of new patient safety programs.

Setting: Osaka University Hospital, a large government-run teaching hospital.

Strategy for change: A voluntary and anonymous web-based incident reporting system was introduced. For the new organizational structure a clinical risk management committee, a department of clinical quality

See end of article for authors' affiliations

Correspondence to: Dr K Nakajima

Department of Clinical Quality Management, Osaka University Hospital, 2-15 Yamadaoka, Suitashi, Osaka 565-0871, Japan; kazuen@hp-cqm. med.osaka-u.ac.jp

Accepted for publication 7 January 2005 management, and area clinical risk managers were established with their respective roles clearly defined to advance the plan-do-study-act cycle and to integrate efforts. For preventive action, alert procedures, staff education, ward rounds by peers, a system oriented approach for reducing errors, and various feedback channels were introduced.

Effects of change: Continuous incident reporting by all hospital staff has been observed since the introduction of the new system. Several error inducing situations have been improved: wrong choice of drug in computer prescribing, maladministration of drugs due to a look-alike appearance or confusion about the manipulation of a medical device, and poor after hours service of the blood transfusion unit. Staff participation in educational seminars has been dramatically improved. Ward rounds have detected problematic procedures which needed to be dealt with.

Lessons learnt: Patient safety programs based on a web-based incident reporting system, responsible persons, staff education, and a variety of feedback procedures can help promote a safety culture, multidisciplinary collaboration, and strong managerial leadership resulting in system oriented improvement.

$\mathrm{P}$

atient safety has attracted intense public attention in Japan since a highly publicized instance of wrong patient surgeries occurred at a university hospital in January 1999. ${ }^{1}$ In response, the Ministry of Health , Labor and Welfare mandated four conditions for tertiary care hospitals providing advanced medical care effective from April 2000, ${ }^{2}$ and for all other hospitals and clinics from October 2002. ${ }^{3}$ These conditions comprised: (1) development of a policy for patient safety; (2) collection of information related to actual and potential harm to patients and others; (3) formation of a committee for the prevention of adverse events; (4) staff education on patient safety. In April 2003 three other requirements were added for tertiary care hospitals: establishment of a division of patient safety, employment of a full time clinical risk manager, and opening of a patient complaint office. ${ }^{3}$ For hospitals not meeting these requirements, the daily reimbursement from the government is reduced by 100 yen (about US\$1) per inpatient. Japanese hospitals, unaccustomed to such requirements, initially faced difficulties establishing a system for patient safety.

\section{OUTLINE OF PROBLEM}

Osaka University Hospital is one of the largest national university hospitals with 1076 beds, 715 physicians, and 12501 annual patient discharges (box 1). In such a large teaching hospital doubts were expressed about the staff's willingness to report problematic practices, interservice communication to resolve problems, and top level managerial leadership for improvement.

Four critical conditions for a well functioning patient safety program were lacking at this time:
(1) an open culture with a consensus among the staff that organizational learning from the failures of individuals is essential for improvement;

(2) a tool for hospital-wide collection of information regarding actual or potential adverse events: only the Nursing Department had been gathering incident reports but had not shared them or resolved common problems with other wards and departments. Incident reports seemed to be perceived as the equivalent of a written apology and thus included few suggestions for preventive measures;

\section{Box 1 Characteristics of Osaka University} Hospital (in 2002)

- A teaching and academic hospital providing tertiary care, including advanced medical care, and authorized by the Ministry of Health, Labor and Welfare.

- 1076 beds, 27 specialties, 715 physicians (including 294 staff physicians), 649 nurses (including part time staff), 333 other employees.

- 12501 annual patient discharges, 574406 annual outpatient visits.

- 6203 annual surgical operations (including 3 cardiac, 8 pulmonary and 11 liver transplantations).

- 863 annual admissions to the Trauma and Acute Critical Care Center, 562 births. 
(3) a lack of clear ideas about the roles of a new committee and department for patient safety as well as ways to involve and integrate frontline people;

(4) a lack of established findings about what type of interventions would be feasible and effective for safer practices.

\section{STRATEGIES FOR CHANGE}

To develop patient safety programs with an open culture, we wanted to introduce a hospital-wide incident reporting system to collect data on variant practices, build an organizational structure for activities aimed at patient safety, and implement staff education and system oriented improvements.

\section{Development of an intranet-based incident reporting system}

In view of findings in the literature that physicians preferred email reporting under non-punitive, confidential, and voluntary conditions, ${ }^{4}{ }^{5}$ we decided to develop an anonymous and blame free web-based incident reporting system. This decision was made at the beginning of 2000 and the system was put into use in July of that year. The system provides easier access for reporting, shorter data entry time, better legibility of reports, and immediate information sharing among persons in charge (fig 1 , box 2 ). ${ }^{6}$

\section{Setting up a new organizational structure for patient safety}

We created a new organizational structure with three new key components: a Clinical Risk Management Committee, a Department of Clinical Quality Management, and area clinical risk managers. We also clarified the functions of the parties responsible for patient safety (fig 2). ${ }^{7}$ The Clinical Risk Management Committee reviews incident reports and plans improvement. The committee comprises 22 multidisciplinary professionals including 15 physicians (a medical vice-director, two departmental chairs, and 12 clinical department representatives), three nurses, a pharmacist, a clinical engineer and three administrators. Incident reports are reviewed for their suitability for case based analysis on a daily basis by a committee member with access to the

\section{Box 2 Advantages of a web-based incident} reporting system

For reporters:

- Easier access through approximately 800 computer terminals in all areas of the hospital.

- Anonymous entry of information and thus reduced psychological resistance to reporting.

- Average completion time for one report only 9 minutes because of many templates with check-off boxes.

- Structured data entry guidance for reporting the facts of an incident, its causes, and preventive actions in the narrative section.

For monitors:

- Legibility of reports.

- Easier and faster monitoring and sharing of information by several members.

- Security of access by responsible persons only using staff identification and a password.

- No additional work of entering paper based information into a database. database rather than at a monthly meeting. Subsequent communications among committee members planning prioritized actions take place mainly through email discussions. The committee has been granted the power to convene multidisciplinary working groups or ask other hospital functional committees for the development and approval of policies and procedures related to patient safety.

The Department of Clinical Quality Management acts on decisions made by the committee to implement plans, investigate in detail the causes of an incident when necessary, and integrate efforts to improve quality of care among relevant areas and staff. It also analyses compilations of incidents for patterns and trends, plans solutions, and develops staff education programs. The department is composed of two full time hospital clinical risk managers with a nursing background and two physicians with other concurrent responsibilities.

The 127 middle managers among physicians, nurses, and other co-medical staff and administrators were appointed as area clinical risk managers in their respective wards or units of responsibility. They oversee the quality of care in their work places, facilitate reporting of incidents, and respond to incidents in their areas by taking corrective actions and communicating as team leaders with patients and family members. Giving a new title to these managers aimed to enhance awareness of their responsibilities for patient safety but did not require much additional work under normal circumstances because these activities are part of their regular commitment to patient care. The department convenes a monthly meeting to educate area clinical risk managers, discuss matters about which they have raised questions, and provide relevant information which they need to convey to their staff.

\section{Education, system improvement and feedback}

The Department of Clinical Quality Management alerted area clinical risk managers through newsletters and a mailing list. These alerts warned about pitfalls in clinical practice and provide information on basic clinical knowledge and rules of which the staff should be aware. In the spring of 2001 the Clinical Risk Management Committee started educational seminars three times a year in an effort to inform all employees of findings based on incident reports as well as to create a hospital-wide safety culture. To increase attendance, a roll call and visible proof of attendance attached to the staff photo ID card were introduced in addition to repeated presentations of the same programs (box 3). To assess whether safer procedures have been put in place and to get area clinical risk managers more involved in patient safety activities, we introduced two types of ward rounds by peers, patrols by the committee members, and mutual ward checks by the area clinical risk managers twice or three times a year. System oriented improvement focusing on root causes of incidents has been emphasized the most among preventive efforts. To encourage the staff to make continuous efforts for patient safety, feedback is made available through newsletters, hospital intranet, clinical risk managers' monthly meetings and their mailing list, and staff seminars. As part of the plan-do-study-act (PDSA) cycle, preventive actions which had been planned by the Clinical Risk Management Committee and implemented by the Department of Clinical Quality Management are monitored through incident reports, ward rounds by peers, and other methods. Changes have then been made when necessary.

\section{EFFECTS OF CHANGE}

Several positive changes have been observed since the strategies were implemented. The web-based incident reporting system has promoted participation of all hospital staff in 


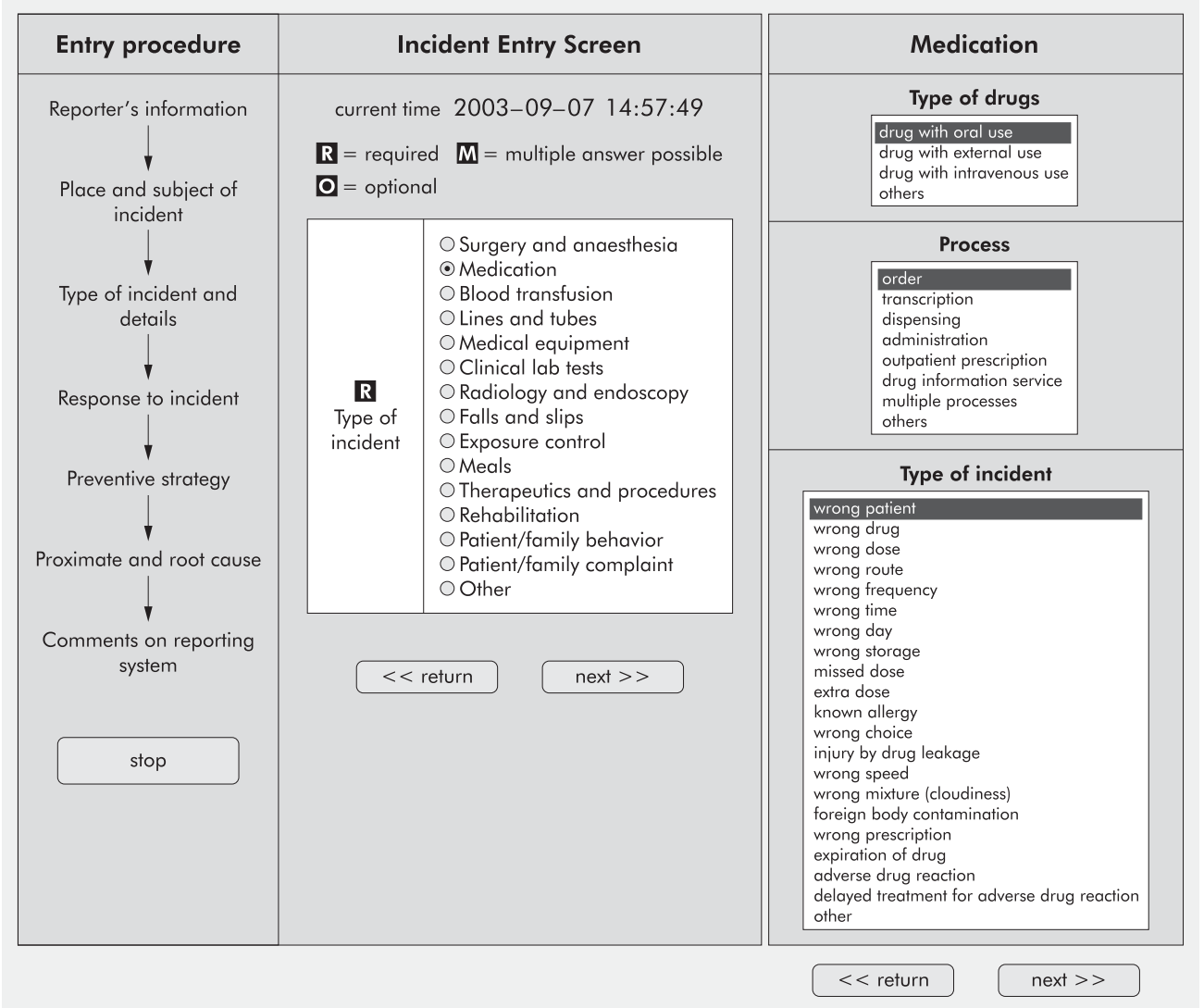

Figure 1 Examples of the web-based incident report entry screen. The reporter clicks a button corresponding to a categorized incident (left). When medication is selected as a type of incident, further detailed information to be input is shown (right).

reporting errors and clarified problems to be resolved. The new organizational structure for patient safety has enabled multidisciplinary efforts to make improvements in a variety of ways.

\section{Continuous reporting by all hospital staff}

The characteristics of the electronic incident reporting system, which include a lower psychological barrier due to anonymous entry as well as easier access and shorter input time for reports, seem to promote reports on a continuous basis from various disciplines. The Nursing Department, which had its own paper based reporting system, at first appeared resistant to the electronic unified system but eventually joined it in June 2001. Before the introduction of the electronic system nurses had submitted 45 reports monthly on average regarding issues specific to nurses' work

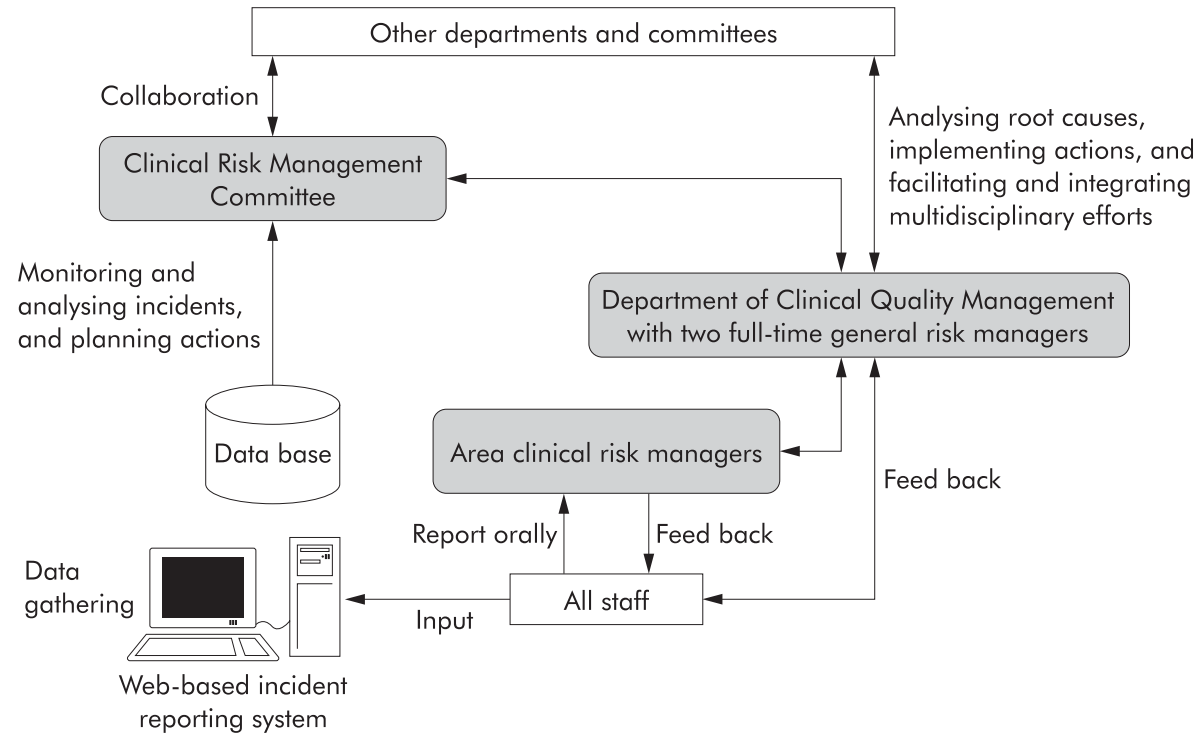

Figure 2 The new organizational structure with the three entities responsible for setting up patient safety programs at Osaka University Hospital. 


\section{Box 3 Tips for successful education programs}

- Provide an annual schedule and secure a place for a large audience.

- Take attendance and provide a proof of attendance visible to other hospital staff and patients.

- Take into account mass media coverage of serious adverse events at other institutions when deciding the timing and contents of seminars in order to enhance their appeal.

- Hold the same seminar several times to give busy employees more opportunities to attend.

- Provide attractive and useful lectures by clinical professionals and administrative personnel both from within and from outside the hospital.

such as drug maladministration, trouble with lines and tubes, slips and falls, and problems related to meals. From this time until 31 March 2004, a total of 6041 reports ( 177 reports per month on average) were submitted (table 1). Incident reports were being continually submitted by hospital employees, with the physicians' monthly contribution accounting for approximately 18 reports ( $10.6 \%$ of all reports). The major types of incidents were medication related $(n=2815,46.6 \%)$, line and tube troubles $(\mathrm{n}=1147,19.0 \%)$, and falls $(\mathrm{n}=826$, $13.7 \%$ ). Major root causes were flawed clinical processes or systems including a lack of verification and poor compliance with protocols $(\mathrm{n}=3446,57.0 \%)$, poor communication among clinicians $(\mathrm{n}=1800,29.8 \%)$ and inadequate supervision $(\mathrm{n}=854,15.1 \%)$.

Such incidents have been used for both hospital-wide and departmental improvement. The nature of incident reporting depended on staff groups or departments. Nurses tended to report any variant care including near misses and errors which did not affect patients. Most of these incidents were orally reported to their supervisors and printed out from the computer terminal for discussion of preventive measures among each ward's nursing staff. On the other hand, physicians tended to report obvious errors that they made and recognized. Physicians' inadequate orders or communication were usually reported by nurses who were involved in some kind of trouble. Physicians did not always report their incidents to their supervisors nor share them at their departmental conferences. They hardly considered problems of clinical judgment, skills for invasive procedures, and teamwork to be incidents that should be shared hospitalwide for patient safety, but considered them matters to be discussed in departmental clinical conferences concerned with quality of care. Hospital divisions such as the operating room and transfusion unit shared the information of incidents with relevant staff and discussed prevention within the divisional committees.

\section{Transient effect of newsletter alerts}

Paper based and web based newsletter alerts were issued and provided to all area clinical risk managers. The Department of Clinical Quality Management staff decide on a topic for a given newsletter and timing of the issue to attract the staff's attention based on a reported incident or media coverage of a serious adverse event, consult experts in the hospital regarding the professional and specialized contents, and then complete the newsletter by adding relevant information.

However, the distribution of newsletters appeared to have a very limited effect on prevention of recurrence of similar
Table 1 Reporters, types and root causes of incidents entered in the web-based incident reporting system from 1 June 2001 until 31 March 2004

\begin{tabular}{lc} 
& \\
& $\mathbf{n}(\%)$ \\
\hline Reporters of incidents & \\
Nurse or midwife & $5115(84.7)$ \\
Physician & $616(10.2)$ \\
Pharmacist & $137(2.3)$ \\
Radiologist & $46(0.8)$ \\
Lab technician & $40(0.7)$ \\
Physical, occupational, or speech therapist & $35(0.6)$ \\
Administrator & $22(0.4)$ \\
Dietician & $5(0.1)$ \\
Others & $25(0.4)$ \\
Total & $6041(100)$ \\
Types of incidents & \\
Medication (ordering, dispensing, administration) & $2815(46.6)$ \\
Lines and tubes & $1147(19.0)$ \\
Falls and slips & $826(13.7)$ \\
Therapeutics and procedures & $197(3.3)$ \\
Clinical laboratory tests & $105(1.7)$ \\
Medical devices and equipment & $95(1.6)$ \\
Meals & $88(1.5)$ \\
Blood transfusion & $87(1.4)$ \\
Radiology and endoscopy & $81(1.3)$ \\
Surgery and anesthesia & $51(0.8)$ \\
Patient or family complaints & $51(0.8)$ \\
Patient or family behavior & $46(0.8)$ \\
Exposure control & $31(0.5)$ \\
Rehabilitation therapy & $23(0.4)$ \\
Others & $398(6.6)$ \\
Total & $6041(100)$ \\
Root causes of incidents (multiple responses) & $3446(57.0)$ \\
Clinical process and system & $1800(29.8)$ \\
Interservice communication & $854(15.1)$ \\
Supervision & $599(9.9)$ \\
Patient education & $301(5.0)$ \\
Medical device and equipment management system & $208(3.4)$ \\
Health information management & $167(2.8)$ \\
Patient management system & $125(2.1)$ \\
Work load and shifts & $113(1.9)$ \\
Hospital information system & $115(1.9)$ \\
Work environment & \\
\hline The web-based incident reporting system was introduced on 1 July 2000 \\
but was first used by all the hospital staff including nurses on 1 June \\
2001. & \\
\hline
\end{tabular}

incidents. Figure 3 shows the number of incidents regarding the collection of a blood sample for blood typing in the sampling tube of another patient which was caused by not checking whether the patient's name on the label of the tube was correct. The fact that a few residents have to draw a large number of blood samples from many inpatients on their wards early in the morning may be a root cause of these incidents. The transfusion unit therefore has introduced a mechanism for detecting such errors which is independent of the voluntary incident reporting system because these are critical errors which can cause incompatibility in blood transfusion. Such errors have occurred a few times a month except in the month that the newsletter came out which alerted the staff to the importance of checking the patient's name on the tube.

This limited effect of such warnings may be caused by the extent of staff education by area clinical risk managers, limited human memory, and frequent changes of physicians in a teaching hospital. This implies that something more than simple warnings is required.

\section{Attendance at seminars}

Changes in the attendance at patient safety seminars are shown in table 2. The number of attendants increased from approximately 50 in 2000 to 524 in 2001 and has been maintained at more than 700 since 2002. 


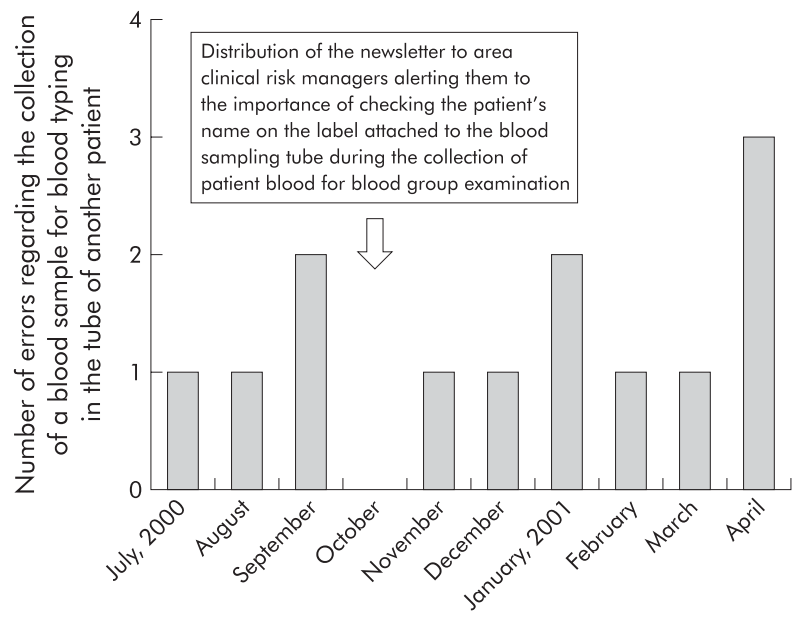

Figure 3 Total number of errors in collecting a blood sample for a blood group examination in the sampling tube of another patient detected by the Transfusion Unit independently from voluntarily reported incidents.

\section{Ward rounds by peers}

The entire medication procedure from ordering to administration as well as blood transfusion procedures, in addition to the contents of all emergency carts and handling of physicians' orally transmitted orders, were checked on all inpatient wards. These checks have uncovered gaps between the practices recommended in the protocols and actual practices. When a significantly variant procedure was observed in a given ward, urgent improvement was recommended within a specified time limit. Such variant procedures included patients' drugs not being kept separate during mixing of intravenous drugs and neither calling out a patient's full name nor confirming it on the medication bags when administering drugs. For benchmarking, a list of the results for all wards without individual ward identification was made available.

\section{Examples of improvements made as a result of reported errors}

The increased reporting of critical incidents has enabled us to make some important improvements in care procedures. The illustrative changes described here concern computer prescription, intravenous administration of a high risk drug, and the manipulation of syringe pumps and blood transfusion.

\section{Change in a drug searching method for computer prescription}

In computer prescribing, one type of critical incident was the erroneous choice of a drug caused by a wrong click on a long list of drugs with similar names shown on one screen. This occurred during a search for an intended drug using the first two letters of a drug's commercial name-for example, incorrect choice of an immunosuppressant (Selcept ${ }^{\circledR}$ ) for a patient requiring an anti-ulcer drug (Selbe $\left.{ }^{\circledR}\right)$, a muscular relaxant $\left(\right.$ Succin $^{\circledR}$ ) instead of a steroid (Saxizon ${ }^{\circledR}$ ). (Note that, in Japanese, the last two drugs have identical spelling for the first syllable). When it was found that a first three letter search resulted in a much higher correct drug specification rate than a first two letter use, ${ }^{8}$ the former was implemented to reduce the likelihood of perceptual confusion. Even after this change, however, several drugs with similar names may still be confused, some of which would be highly harmful to a patient when wrongfully prescribed such as Taxotel ${ }^{\circledR}$ (docetaxel hydrate) and Taxol ${ }^{\circledR}$ (paclitaxel). For these drugs an additional warning is displayed when one of them is selected.

\section{Elimination of a "look alike" drug}

To eliminate errors in drug administration, both the Pharmaceutical Committee and Clinical Risk Management Committee have decided to delete 10\% lidocaine from the hospital drug list in April 2003. This action was taken in response to a tragic incident at a certain university hospital where a physician and a nurse erroneously administered $10 \%$ lidocaine by intravenous push instead of by drip infusion, even though they intended to administer $2 \%$ lidocaine by intravenous push. ${ }^{9}$ A major cause of this incident was the similar appearance of the two drug ampoules. The committee's decision was based on the result of discussions among the hospital's cardiologists, cardiovascular surgeons, and intensive care physicians. The discussion dealt with the question of whether patients can be treated adequately without a higher concentration of lidocaine when those with severe heart failure and requiring less volume overload developed ventricular arrhythmias. After this action was decided upon, three more cases of identical medication errors were reported in the mass media. The Japanese Council for circulation-related societies in October 2003 and the Japanese circulation-related societies in June 2004 issued a warning that $10 \%$ lidocaine should not be placed in general wards and emergency carts. ${ }^{10}{ }^{11}$

\section{Improvement in the operation of syringe pumps}

Collaboration with the Department of Medical Engineering, which has not been established yet in all Japanese university hospitals, has led to improvements in error inducing conditions where nurses mistakenly set the total volume instead of the speed when using a syringe pump for administering high risk drugs. This error was related to the pump's design where one button has two critically different functions, one to set the speed per hour and the other the total volume of the fluid to be administered. Except in two wards, the function of the total volume in approximately 200 syringe pumps of this type was deactivated without waiting for the expiration of these devices or for the purchase of a better model.

Table 2 Number attending patient safety seminars

\begin{tabular}{llll}
\hline & June & October & February \\
\hline $\begin{array}{lll}\text { Attendants } \\
\text { Year }\end{array}$ & All employees & Clinicians & Clinicians \\
2000 & & & Approx $50(1)$ \\
2001 & $513(4)$ & $363(2)$ & $383(2)$ \\
2002 & $703(4)$ & $233(1)$ & $570(2)$ \\
2003 & $738(4)$ & $235(1)$ & $500(2)$ \\
2004 & $774(4)$ & \\
\hline \multicolumn{2}{l}{ Numbers in parentheses show number of sessions per seminar. } \\
\hline
\end{tabular}




\section{Error detecting system for blood transfusion}

To prevent erroneous typing for blood transfusion the transfusion unit implemented three effective procedures. ${ }^{12}$ The first is blood sampling on two different occasions for the pre-transfusion test to avoid erroneous collection in another patient's sample tube. The second is an automatic checking system with a barcode reader to detect the selection of a wrong blood product from the refrigerator on a ward. The third is the use of a large sign showing the patient's name and blood type hung on the intravenous drip stand for the final check. However, the most vulnerable process-where physicians had to perform blood group examinations, antibody screening and compatibility tests by themselves when after-hours transfusion was needed-could not be changed by the transfusion unit alone. With strong leadership by a medical director, a system change entailing training of additional laboratory technicians and the purchase of an automated machine was introduced after a fatal incident resulting from the inexperience of residents which occurred at another hospital. ${ }^{13}$

\section{Development of the PDSA cycle}

To ensure progress in the PDSA cycle, the Department of Clinical Quality Management has monitored the effects of preventive actions and brought up issues with the Clinical Risk Management Committee where action needed to be taken against incidents that occurred repeatedly in spite of the implementation of preventive measures. When erroneous ordering of Taxol $^{\circledR}$ instead of Taxotel ${ }^{\circledR}$ occurred in a university hospital with the same computer prescription system as that used at our institution which only displayed the warning: "This drug is antineoplastic. Are you sure you want to prescribe it?," we immediately decided to display both the commercial and generic names for such drugs. ${ }^{14}$ Repeated incidents of mistaken patient identity despite the rule to check both patient identity and relevant procedures led to a decision by the committee to put a name tag on the wrist of all inpatients. In addition, the committee is currently planning to introduce a computerized system for checking and recording patient identity and relevant procedures to compensate for the limited reliability and accuracy of human checking.

\section{LESSONS LEARNT AND FURTHER STEPS}

The web-based incident reporting system is currently recognized as a useful tool for patient safety in individual hospitals ${ }^{15}{ }^{16}$ as well as at the national level. ${ }^{17}{ }^{18}$ The use of a computerized system in limited settings such as intensive care units succeeded in involving physicians in reporting to a greater extent, where they accounted for more than $20 \%$ of the total number of reports. ${ }^{19}{ }^{20}$ Reported incidents have led professional groups to take action, including making recommendations for restrictions on storage areas for high risk drugs and the establishment of a Department of Clinical Engineering for the centralized management of medical devices. ${ }^{10} 1121-23$ An Australian hospital found that the number of critical adverse events detected by medical record review was reduced after the introduction of patient safety programs including incident reporting. ${ }^{24}$ Top level managerial decision making is emphasized for system oriented improvements involving considerable budgets. ${ }^{25}$

Our experience shows that adoption by the staff of reporting incidents and a faster response to problems brought to light as a result of such reporting-which were the barriers that we faced in introducing the patient safety programscan be resolved by the web-based incident reporting system which streamlines the process of reporting and information sharing. Physicians' reports accounted for a slightly higher percentage than the average of 79 Japanese university

\section{Key messages}

- The web-based incident reporting system is an effective tool with advantages suitable for promoting an open culture for patient safety.

- A committee responsible for implementation of the system, full time hospital clinical risk managers, and area clinical risk managers are essential for collaboration to enhance patient safety.

- A system oriented approach should be established to detect, absorb, and avoid errors in addition to issuing warnings and providing staff education.

- Feedback should be provided in a variety of ways to encourage continuous improvement and to foster a safety culture.

- The combination of all these key points appears to eliminate or reduce major barriers to the creation and establishment of patient safety programs in larger hospitals such as staff resistance, poor inter-service communication, and inadequate managerial leadership.

hospitals $\left(8.3 \%\right.$ in 2003) ${ }^{26}$ where paper based information gathering was still the major procedure. Multidisciplinary efforts for the implementation of improvements and safer practices by the staff also represented problems which had to be overcome. The establishment of a department responsible for patient safety with at least one full time hospital clinical risk manager is considered indispensable for staff education, gathering of relevant information, and the preparation of proposals for submission to the committee and of intrahospital communication for action. Safer practices in individual hospital areas may be influenced by the attitude and behavior of the area clinical risk managers so that their education and involvement is a key to the efficient functioning of these programs. The creation of a safety culture, the development of which seems to be indicated by the continuous nature of incident reporting and increased attendance at seminars, can be achieved by not only repeated emphasis on the importance of learning but also by providing information in various ways and forms on changes and their effects.

Further actions will focus on improvement of poor medical practices related to erroneous clinical judgment, inadequate technical skills, and teamwork which are difficult to identify in this incident reporting system. We will also examine any overall measurable effect of these efforts on patient safety in addition to qualitative analyses shown in this study. Besides intra-hospital efforts, external factors such as health care policies supporting health care institutions' investment in safer practices and legal protection for documents relevant to peer review activities are needed to promote patient safety and quality of care.

\section{ACKNOWLEDGEMENTS}

The authors thank Ms Kazuyo Hatta and Ms Noriko Okamoto for their support in providing detailed information on incident analyses and preventive actions, and Dr Robert B Leflar for his professional advice from legal and patient points of view.

\section{Authors' affiliations}

K Nakajima, Department of Clinical Quality Management, Osaka University Hospital, Japan

Y Kurata, Department of Blood Transfusion, Osaka University Hospital, Japan

H Takeda, Department of Clinical Quality Management, Osaka University Hospital, Japan 


\section{REFERENCES}

1 Anon. Blundering doctor fined for performing wrong operations. Mainichi Daily News. Available at hitp://www12.mainichi.co.jp/news/mdn/searchnews/923706/Yokohama20City20University20Hospital-0-3.html.

2 Ministry of Health, Labour and Welfare. Ordinance partially amending Ministerial Ordinances on Health Services Law No. 7 of 31 January 2001 (in Japanese).

3 Ministry of Health, Labour and Welfare. Ordinance partially amending Ministerial Ordinances on Health Services Law No. 101 of 30 August 2002 (in Japanese).

4 O'Neil AC, Petersen LA, Cook EF, et al. Physician reporting compared with medical record review to identify adverse medical events. Ann Intern Med 1993; 119:370-6.

5 Cohen MR. Why error reporting systems should be voluntary. BMJ 2000;237:728-9.

6 Nakajima K, Shigeki K, Matsumura Y, et al. Operation and effects of computerized incident reporting system for prevention of adverse medical events (in Japanese). Jpn J Med Inf 2001;21:77-82.

7 Takeda H, Matsumura Y, Nakajima K, et al. Health care quality management by means of an incident report system and an electronic patient record system. Int J Med Inf 2003;69:285-93.

8 Furukawa H, Tsuchiya F, Onishi H, et al. Investigation of feasibility of physician order entry system for risk management strategy related to medication errors (in Japanese). Jpn J Med Inf 2001;21:69-76.

9 Anon. Patient death due to medication overdose (in Japanese). Asahi Newspaper (morning edition), 11 December 2002, 39.

10 Japanese Council for Quality Health Care. Urgent recommendation on the storage of $10 \%$ lidocaine and ampoule-type potassium chloride concentrate solution (in Japanese). 28 October 2003.

11 Japanese Circulation-Related Societies. Urgent notice on the storage of $10 \%$ lidocaine and ampoule-type potassium chloride concentrate solution (in Japanese). 25 June 2004.
12 Kurata $\mathrm{Y}$, Kiyokawa $\mathrm{T}$, Aochi $\mathrm{H}$, et al. Report on $\mathrm{ABO}$-incompatible transfusions at 12 university hospitals in the Kinki District (in Japanese). Jpn J Transfus Med 2000;46:17-22.

13 Anon. Trainees botch transfusion tests. Mainichi Daily News. Available at http://www12.mainichi.co.jp/news/mdn/search-news/923706 transfusion20tests-0-3.html.

14 Anon. Wrong medication killed a cancer patient; Taxote ${ }^{\circledR}$ ordered instead of Taxo $^{\circledR}$ in computer prescription (in Japanese). Asahi Newpaper (morning edition), 22 October 2003, 39

15 Maass G, Cortezzo M. Computerizing incident reporting at a community hospital. Jt Comm J Qual Improv 2000;26:361-73.

16 Mekhiian HS, Bently TD, Ahmad A, et al. Development of a web-based event reporting system in an academic environment. JAMIA 2004;11:11-18.

17 Australian Patient Safety Foundation. Effective incident monitoring: collect, analyse, learn. 2001:1-8.

18 NHS National Patient Safety Agency. Newsline, August 2003 (www.npsa.nhs.uk).

19 Busse DK, Wringht DJ. Classification and analysis of incidents in complex medical environments. Topics in Health Information Manage 2000;20:1-11.

20 Wu A, Pronovost P, Morlock L. ICU incident reporting systems. J Crit Care 2002;17:86-94.

21 NHS National Patient Safety Agency. Patient Safety Alert (on potassium chloride concentrate solution), 23 July 2002 (www.npsa.nhs.uk).

22 NHS National Patient Safety Agency. Safer practice notice (on infusion devices), 20 May 2004 (www.npsa.nhs.uk).

23 Joint Commission on Accreditation of Healthcare Organizations. Sentinel Event Alert (www. jcaho.org).

24 Wolff AM, Bourke J, Campbell IA, et al. Detecting and reducing hospital adverse events: outcomes of the Wimmera clinical risk management program. Med J Aust 2001;174:621-5.

25 Leape LL, Bate DW, Cullen DJ, et al. System analysis of adverse drug events. JAMA 1995;274:35-43.

26 Anon. Quality differences among 79 university hospitals (in Japanese). Asahi Newpaper (evening edition), 13 July 2004, 1. 